\title{
An Artificial Neuron with Quantum Mechanical Properties
}

\author{
Dan Ventura and Tony Martinez \\ Neural Networks and Machine Learning Laboratory (http://axon.cs.byu.edu) \\ Department of Computer Science \\ Brigham Young University, Provo, Utah 84602 USA \\ dan@axon.cs.byu.edu,martinez@cs.byu.edu
}

\begin{abstract}
Quantum computation uses microscopic quantum level effects to perform computational tasks and has produced results that in some cases are exponentially faster than their classical counterparts. Choosing the best weights for a neural network is a time consuming problem that makes the harnessing of this "quantum parallelism" appealing. This paper briefly covers necessary high-level quantum theory and introduces a model for a quantum neuron.
\end{abstract}

\section{Introduction}

The field of artificial neural networks has at least two important goals: (A) creation of powerful artificial problem solving systems and (B) furthering understanding of biological neural networks including the human brain. Much effort has been made in both areas and some progress has been realized. The field of quantum computation [1], which has been completely unrelated to that of neural networks until very recently, applies ideas from quantum mechanics to the study of computation and has made interesting progress. Most notably, quantum algorithms for prime factorization and discrete logarithms have recently been discovered that provide exponential improvement over the best known classical methods [2]. Recently some work has been done in the area of combining classical artificial neural networks with ideas from the field of quantum mechanics in pursuit of goal (A).

It is the purpose of this paper to further this pursuit of goal (A), that is, to show the usefulness of some ideas from the field of quantum mechanics (in particular those of linear superposition and coherence/decoherence) to that of artificial neural networks in order to improve neural networks' abilities as problem solving systems. Our approach is to introduce a mathematical model of an artificial neuron with quantum mechanical properties that allow it to discriminate linearly inseparable problems using only linear thresholding as its activation function.

\section{Some Quantum Ideas}

Quantum mechanics is in many ways extremely counterintuitive and yet it has provided us with perhaps the most accurate theory (in terms of predicting experimental results) ever devised by science. The theory is well established and is covered in its basic form by many textbooks (see for example [3]). Several ideas from this theory that are necessary for the following presentation must be briefly mentioned.

Linear superposition is closely related to the familiar mathematical principle of linear combination. For example, in a vector space with bases $\boldsymbol{x}$ and $\boldsymbol{y}$, any vector $\boldsymbol{v}$ can be defined as $\boldsymbol{v}=a \boldsymbol{x}+b \boldsymbol{y}$. In some sense $\boldsymbol{v}$ can be thought of as being both $\boldsymbol{x}$ and $\boldsymbol{y}$ at the same time. In quantum mechanics, this principle actually applies to physical variables in physical systems. The vector space is generalized to a Hilbert space whose bases are the classical values normally associated with the system. For example, the position of an electron orbiting a nucleus is usually a superposition of all possible positions in 3-d space, where each possible position is a basis state for the Hilbert space and has a finite probability of being the actual position. One of the most counterintuitive aspects of quantum theory is this -- at the quantum or microscopic level, the electron is not in any one position in an orbit, but it is in a superposition of all of them at once. In some sense it is in all positions at the same time. However, at the macroscopic or classical level, the location of the electron is a single definite position in 3-d space. This apparent contradiction is still not fully understood, but it is explained as follows. A quantum mechanical system remains in a superposition of its basis states until it interacts with its environment.

Coherence/decoherence is related to the idea of linear superposition. A quantum system is said to be coherent if it is in a linear superposition of its basis states. As mentioned above, a result of quantum mechanics is that if a system that is in a linear superposition of states is observed or interacts in any 
way with its environment, it must instantaneously choose one of those states and "collapse" into that state and that state only. This collapse is called decoherence and is governed by the wave function $\Psi$. Just as the basis states of the Hilbert space have a physical interpretation (as the classical values associated with a system), so too does the amplitude of the wave function $\Psi$ describing the system. This wave function actually represents the probability amplitudes (in the complex domain) of all bases (possible positions, etc.) such that the probability for a given basis (position, etc.) is given by $|\Psi|^{2}$.

Operators are a mathematical formalism used to describe how one wave function is changed into another. They are analogical to matrices operating on vectors. Using operators, an eigenvalue equation can be written $\hat{A} \phi_{i}=a \phi_{i}$. The solutions $\phi_{i}$ to such an equation are called eigenstates and are the basis states of a Hilbert space. In the quantum formalism, all properties (position, momentum, spin, etc.) are represented as operators whose eigenstates are the classical values normally associated with that property.

\section{Related Work}

To date, very little has been done in combining the fields of quantum mechanics and neural networks. However, a few notable exceptions do exist. Perus has published an interesting set of mathematical analogies between the quantum formalism and neural network theory [4]. Menneer and Narayanan have proposed a model weakly inspired (their term) by the "many worlds" interpretation of quantum mechanics in which a network is trained for each instance in the training set and the final network is a superposition of these [5]. Finally, Behrman et. al. have developed a novel approach to implementing a quantum neural network using quantum dots [6]. It uses a quantum dot for each input and the system is allowed to evolve quantum mechanically through time while being observed (and thus forced to decoher) at fixed time intervals. Interestingly, the different time slices act as the neurons in a hidden layer of a neural network -- the more time slices, the more hidden layer neurons. Perhaps most notably, Behrman et. al. have actually implemented this quantum dot neural network and give results for its learning several two input boolean functions.

\section{Quantum Neuron}

The simplest classical artificial neuron is the perceptron that takes as input $n$ bipolar (or binary) values, $\left\{i_{j}\right\}$. It is defined as a weight vector $\boldsymbol{w}=\left(w_{l}\right.$, $\left.w_{2}, \ldots, w_{n}\right)^{T}$, a threshold $\theta$ and an output function $f$ where

$$
f=\left\{\begin{aligned}
1 & \text { if } \sum_{j=1}^{n} w_{j} i_{j}>\theta \\
-1 & \text { otherwise }
\end{aligned}\right.
$$

This neuron model is well understood, but it is mentioned for several reasons. First, it cannot solve problems that are not linearly separable, and second, though this classical perceptron is extremely simple and well understood, this will not be the case for its quantum counterpart and thus it is important to start with the very simplest concepts as quantum ideas are incorporated.

We now define a simple quantum analog to the perceptron, which also takes as input $\left\{i_{j}\right\}$. It is similar in all respects to the classic perceptron except that the single weight vector $\boldsymbol{w}$, is replaced by a wave function, $\Psi(\boldsymbol{w}, t)$ in a Hilbert space whose basis states are the classical weight vectors. This wave function represents the probability amplitude (in general this is a complex wave as opposed to a real one) for all possible weight vectors in weight space together with the normalization condition that for any time $t$

$$
\int_{-\infty}^{\infty}|\Psi|^{2} d \boldsymbol{w}=1
$$

Thus, the weight vector of the perceptron is replaced with a quantum superposition of many weight vectors which on interaction with its environment will decoher into one classical weight vector, according to the probabilities given by $|\Psi|^{2}$.

For example, consider the one-input, one-output bipolar function that inverts its input (NOT). For convenience the weights will be bounded such that

$$
-\pi \leq w_{j} \leq \pi
$$

In order for a quantum neuron to learn this function, $\Psi(\boldsymbol{w}, t)$ and $\theta$ must be found. We assume that $\Psi$ is time-invariant and concentrate only on $w$, which is in this case a one-dimensional vector. Because of the strict bounds given in (3), finding $\Psi$ is equivalent to solving the one-dimensional rigid box problem common to most elementary treatments of quantum mechanics, whose solutions are of the form

$$
\Psi\left(w_{0}\right)=A \sin \left(\frac{n \pi}{a} w_{0}\right) .
$$


Here $A$ is a normalization constant that can be found using (2), $n=1,2,3, \ldots, w_{0}$ is the single element of $\boldsymbol{w}$, and $a$ is the width of the box (in general this is $2 \pi$, but the width may be altered to be smaller).

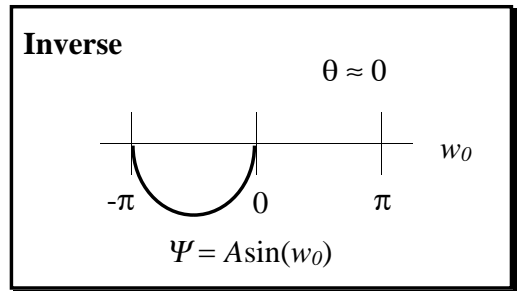

Figure 1. Solutions for one-dimensional NOT function

Figure 1 shows a graph of one $\Psi$ for the one-input bipolar NOT function, and for comparison figure 2 shows a solution for another one-input bipolar function, TRUE (the extra $\pi$ term shifts the function, which is necessary since the general solution (4) assumes for convenience that the left hand side of the box occurs at 0 ). To understand what these graphs represent, it must be realized that they are graphs in weight space and that $\Psi$ is the probability amplitude for a given weight vector $\boldsymbol{w}$. In a quantum neuron the weight vectors exist in coherent superposition of all possible classical weight vectors in weight space with non-zero probability amplitude.

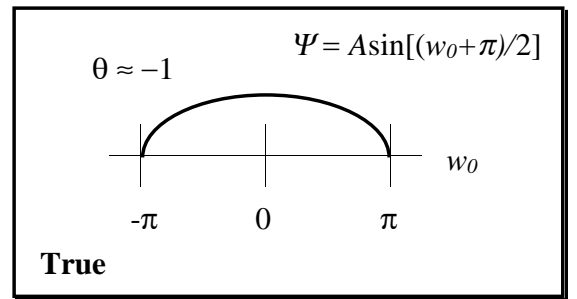

Figure 2. Solutions for one-dimensional TRUE function

When the superposition of weight vectors interacts with its environment (for example when it encounters an input) it must decoher into one basis state -- a classical weight vector within the bounds enforced in (3) -- and this decoherence occurs with probability governed by $|\Psi|^{2}$. Note that in the case of these simple functions, no matter what weight vector is chosen at decoherence, it will result in the correct output.

Now consider the more complicated two-input XOR problem, which is of course, not linearly separable. Using a similar argument for that of the one-dimensional case, it is not surprising to find that the solutions to problems with two-dimensional weight spaces are equivalent to those of the two dimensional rigid box

$$
\Psi\left(w_{0}, w_{1}\right)=A \sin \left(\frac{n_{w 0} \pi}{a} w_{0}\right) \sin \left(\frac{n_{w 1} \pi}{a} w_{1}\right),
$$

where the variables and constants have the same meanings as in (4) with the added note that now there are two different $n$ 's, one for each weight.

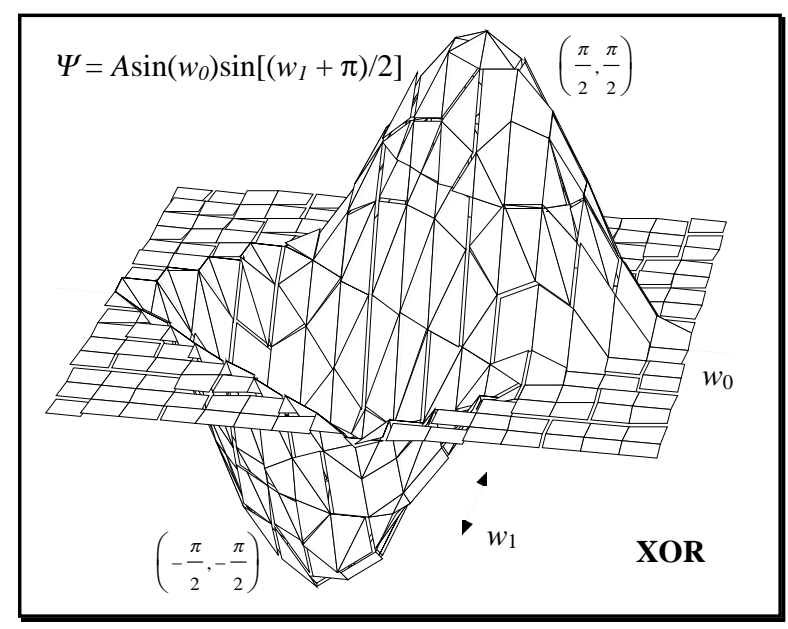

Figure 3. Plot of $\Psi$ for the XOR problem

Figure 3 shows a contour plot of one such solution, rotated by $45^{\circ}$. Note again that this is a graph of $\Psi$, the probability amplitude, whereas the probability, $|\Psi|^{2}$, is what is really important.

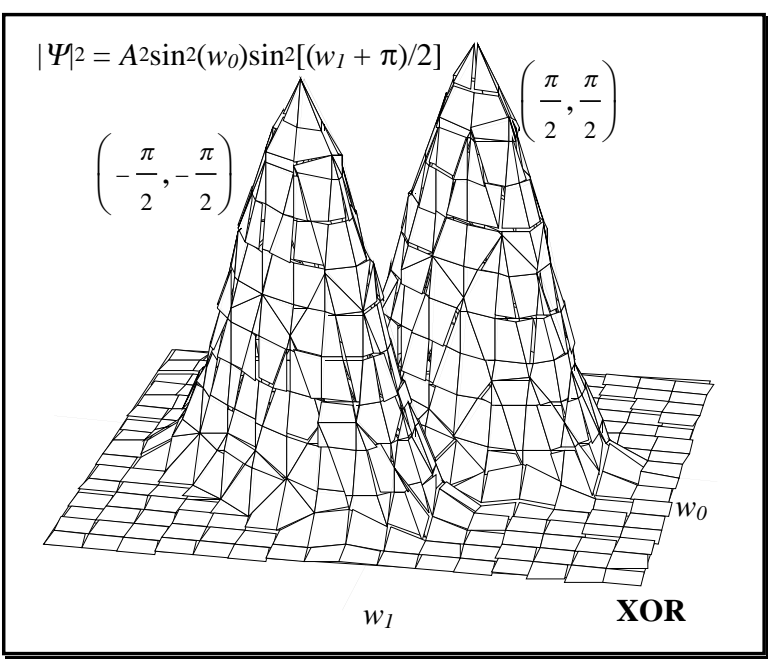

Figure 4. Plot of $|\Psi|^{2}$ for the XOR problem 
Also notice that its contour plot (figure 4) gives two solutions with maximal probability, at $(\pi / 2, \pi / 2)$ and at $(-\pi / 2,-\pi / 2)$. It is also important to note that the nodal line that exists at $\mathrm{w}_{0}=-\mathrm{w}_{1}$ in figure 3 ensures that no solutions exist on this line which must be the case in order to learn the function. Finally note that since the quantum neuron maintains a coherent superposition of weights, it can solve non-linearly separable problems.

Of course, these examples are simple and their solutions are simple. Even in the general case however, the solutions will always be equivalent to those of an $n$ dimensional rigid box so that $\Psi$ will always be of the general form introduced above. This need not be true, however, if other physical models, such as the non-rigid box, simple harmonic oscillator, hydrogen atom, etc. are be considered. Further, the time variable has not been incorporated into the equations. Another important topic is training of the neuron, which entails changing the wave function $\Psi$. Since $\Psi$ is governed by the (time-independent for now) Schrödinger equation,

$$
\nabla^{2} \Psi=\frac{2 m}{\hbar^{2}}[U-E] \Psi,
$$

note that the only variable that may be changed is the potential, $U$. Therefore $\Psi$ may be changed by changing $U$.

The $n$ 's introduced in (4) and (5) are termed quantum numbers and play an important role in quantum mechanics. Further investigation of their role with regards to quantum neurons is needed. A series of classification operators that ensures appropriate wave function decoherence is also required. For example, in the XOR problem, two of the possible input patterns, $(-1,1)$ and $(1,-1)$, are correctly classified using any of the superposed weight vectors; however, for the other two input patterns, $(-1,-1)$ and $(1,1)$, this is not the case. The necessary operators for the quantum neuron will be analogical to a matrix form of the input vector. The operator associated with the input vector $(-1,-1)$ must greatly decrease the probability amplitude (and thus the probability) for all the negative weight vectors and correspondingly increase the amplitudes for the positive weight vectors. The operator associated with the input vector $(1,1)$ must do the opposite.

\section{Concluding Remarks}

Though many of these topics are beyond the scope of this paper, it has been demonstrated that the application of quantum mechanical ideas to the field of neural networks is a fertile area for further research. This includes development of a learning algorithm for the quantum neuron, further investigation of the classification operators, and theoretical analysis of the quantum neuron's capabilities. Further, the idea of linear superposition may be applied not only to the weight vector of a neuron, but also to its inputs, its output, and its activation function, among other things. See [7] for an application to the problem of choosing useful features from the exponentially large set of possibilities. Also, other quantum mechanical concepts such as the quantum nature of energy, spin, momentum, etc. and EPR phenomenon may find application as this field is explored further.

\section{References}

[1] Brassard, Gilles, "New Trends in Quantum Computation", 13th Symposium on Theoretical Aspects of Computer Science, 1996.

[2] Shor, Peter W., "Polynomial-Time Algorithms for Prime Factorization and Discrete Logarithms on a Quantum Computer", to appear in the SIAM Journal of Computing, 1996.

[3] Taylor, John R. and Zafiratos, Chris D., Modern Physics for Scientists and Engineers, Prentice Hall, Englewood Cliffs, New Jersey, 1991.

[4] Perus, Mitja, "Neuro-Quantum Parallelism in BrainMind and Computers", Informatica, vol. 20, pp. 173-183, 1996.

[5] Menneer, Tammy and Narayanan, Ajit, "Quantuminspired Neural Networks", technical report R329, Department of Computer Science, University of Exeter, Exeter, United Kingdom, 1995.

[6] Behrman, E. C., Steck, J. E., and Skinner, S. R., "A Quantum Dot Neural Network", preprint submitted to Physical Review Letters.

[7] Ventura, Dan and Martinez, Tony, "Application of Quantum Mechanical Properties to Machine Learning", submitted to the International Conference on Machine Learning, 1997. 